\title{
AN APPROXIMATION THEOREM FOR INFINITE GAMES ${ }^{1}$
}

\author{
MICHAEL ORKIN
}

\begin{abstract}
We consider infinite, two person zero sum games played as follows: On the $n$th move, players $A, B$ select privately from fixed finite sets, $A_{n}, B_{n}$, the result of their selections being made known before the next selection is made. A point in the associated sequence space $\Omega=\prod_{n=1}^{\infty}\left(A_{n} \times B_{n}\right)$ is thus produced upon which $B$ pays $A$ an amount determined by a payoff function defined on $\Omega$. We show that if the payoff functions of games $\left\{G_{n}\right\}$ are upper semicontinuous and decrease pointwise to a function which is the payoff for a game, $G$, then $\operatorname{Val}\left(G_{n}\right) \downarrow \operatorname{Val}(G)$. This shows that a certain class of games can be approximated by finite games. We then give a counterexample to possibly more general approximation theorems by displaying a sequence of games $\left\{G_{n}\right\}$ with upper semicontinuous payoff functions which increase to the payoff of a game $G$, and where $\operatorname{Val}\left(G_{n}\right)=0$ for all $n$ but $\operatorname{Val}(G)=1$.
\end{abstract}

Introduction. Infinite games with imperfect information have been studied by several writers, notably Blackwell [1], [2], Gillette [3], Milnor and Shapley [4].

Before proceeding with the main result we will introduce notation and describe the structure of these games.

Let $\left\{A_{n}\right\},\left\{B_{n}\right\}$ be sequences of nonempty finite sets. Let $Z_{n}=A_{n} \times B_{n}$ and let $\Omega$ be the space $\prod_{n=1}^{\infty} Z_{n}$ of infinite sequences $\omega=\left(z_{1}, z_{2}, \cdots\right)$ where $z_{n} \in Z_{n}$. Let $X=\left\{\left(z_{1}, z_{2}, \cdots, z_{n}\right) \mid z_{i} \in Z_{i}, n=1,2, \cdots\right\}$ be the set of finite starting sequences or partial histories.

Suppose $f$ is a bounded Baire function on $\Omega$ (with respect to the product topology). Then $f$, called a payoff function, defines a zero-sum two person game $G_{f}$, played as follows:

First, player $A$ selects $a_{1} \in A_{1}$ while player $B$ simultaneously selects

Received by the editors September 29, 1971.

AMS 1970 subject classifications. Primary 90D05; Secondary 60G45.

Key words and phrases. Imperfect information, zero sum two person game, infinite game, random strategy.

1 This paper is part of the author's doctoral dissertation at the University of California, Berkeley, and was prepared with the partial support of U.S. Air Force Grant AF-AFOSR-1312-67 and U.S. Office of Naval Research Contract NONR N00014-66C0036.

(c) American Mathematical Society 1972 
$b_{1} \in B_{1}$. The result, $z_{1}=\left(a_{1}, b_{1}\right) \in Z_{1}$, is announced to both players, upon which $A$ selects $a_{2} \in A_{2}$ while $B$ is selecting $b_{2} \in B_{2}$, etc. The result of this infinite sequence of moves is a point $\omega=\left(z_{1}, z_{2}, \cdots\right) \in \Omega$ and $B$ pays $A$ the amount $f(\omega)$. For any partial history $x \in X$ we can define a subgame of the original game (usually referred to as the original game, "starting from $x$ ") by having the players play as above except redefining the payoff function as $f_{x}(\omega)=f(x \omega)$.

A strategy $\alpha(\beta)$ for $A(B)$ gives for each partial history $x$ (of length $n$, say) a probability distribution on $A_{n+1}\left(B_{n+1}\right)$ with the stipulation that if the current position is $x, A(B)$ will make his next choice according to $\alpha(\beta)$. A pair of strategies, $(\alpha, \beta)$ defines a probability distribution, $P_{\alpha \beta}$ on $\Omega$ and, hence, an expected payoff to $A$ in $G_{f}$ when $A$ uses $\alpha$ and $B$ uses $\beta$ :

$$
E(f, \alpha, \beta)=\int f(\omega) d P_{\alpha \beta}(\omega)
$$

(We will usually omit the $\alpha, \beta$ from the notation when it is clear what is happening.)

The lower and upper values of $G_{f}$ are, respectively,

$$
L\left(G_{f}\right)=\sup _{\alpha} \inf _{\beta} E(f, \alpha, \beta), \quad U\left(G_{f}\right)=\inf _{\beta} \sup _{\alpha} E(f, \alpha, \beta) .
$$

It is always true that $L\left(G_{f}\right) \leqq U\left(G_{f}\right)$; if $L\left(G_{f}\right)=U\left(G_{f}\right)$, this common value is called the value of $G_{f}$ and will be denoted by $\operatorname{Val}\left(G_{f}\right)$.

Finally, a payoff function $f$ is called upper (lower) semicontinuous if $\left(\omega_{n} \rightarrow(\omega) \Rightarrow \lim \sup _{n} f\left(\omega_{n}\right) \leqq f(\omega)\left(\lim _{n} \inf _{n} f\left(\omega_{n}\right) \geqq f(\omega)\right)\right.$.

The result of [5] we will use is as follows. Let $M$ be compact, $N$ any space, $f$ defined on $M \times N$ which is concave-convexlike. If $f(\mu, v)$ is u.s.c. in $\mu$ for each $\nu$, then $\sup _{\mu} \inf _{v} f=\inf _{v} \sup _{\mu} f$. We show how to apply this to the present situation: The space of plays, $\Omega$, and the set of strategies for each player gives rise to a product of compact spaces, $\Omega_{A}^{*} \times \Omega_{B}^{*}$, where $\Omega_{A}^{*}=\prod_{n=1}^{\infty} A_{n}^{*}, \Omega_{B}^{*}=\prod_{n=1}^{\infty} B_{n}^{*}$. We define $A_{n}^{*}, B_{n}^{*}$ as follows: If $\alpha$ is a strategy for player $A$, the corresponding member of $\Omega_{A}^{*}$ is a sequence $\left(\alpha_{1}, \alpha_{2}, \cdots, \alpha_{n}, \cdots\right)$, where $\alpha_{n} \in A_{n}^{*}$ is a finite list of probabilities on $A_{n}$, one for each possible past history (the list is finite since the sets $A_{n}, B_{n}$, $n=1,2, \cdots$, are finite). $B_{n}^{*}$ is defined analogously. The corresponding product topology makes $\Omega_{A}^{*}, \Omega_{B}^{*}$ compact. If $f$ is a payoff on $\Omega$, we get a corresponding payoff $f^{*}$ on $\Omega_{A}^{*} \times \Omega_{E}^{*}$ by defining $f^{*}(\alpha, \rho)=E(f, \alpha, \beta)$. If $f$ is u.s.c. on $\Omega$, so is $f^{*}$ on $\Omega_{A}^{*} \times \Omega_{b 3}^{*}$ (in the product topology); also $f^{*}$ is linear, so [5] applies. It is easily seen that $\sup _{\alpha} \inf _{\beta} f^{*}=\inf _{\beta} \sup _{\alpha} f^{*}$ implies the game with payoff $f$ has a value, so [5] gives us that games with u.s.c. payoffs have a value. 
We shall now prove the main result, namely

THEOREM 2.1. Suppose $G_{f_{n}}$ are games with upper semicontinuous payoff functions $f_{n}$, where the $f_{n}$ are (pointwise) nonincreasing, $f_{n} \downarrow f$ (which is, therefore, also upper s.c.). Then $\operatorname{Val}\left(G_{f}\right)=\lim _{n} \operatorname{Val}\left(G_{f_{n}}\right)$.

We first prove a lemma.

LemMa 2.1. Suppose $f_{n}, f$ are as above. For any partial history $x$, let $m_{x}=\lim _{n} \operatorname{Val}_{x}\left(G_{f_{n}}\right)$, where $\operatorname{Val}_{x}\left(G_{f_{n}}\right)$ means the value of the game with payoff $f_{n}$, starting from $x$. (The value of games with upper s.c. payoff function exists, by [5].) Let $G_{x}^{*}$ be the one move game which starts at $x$ and has payoff $g=m_{y}$ if $y$ is the next position hit. Claim $\operatorname{Val}\left(G_{x}^{*}\right) \geqq m_{x}$.

Proof of Lemma. We will show by contradiction that for fixed $\varepsilon>0, A$ can play in $G_{x}^{*}$ to guarantee that $E(g) \geqq m_{x}-\varepsilon$. Assume not; then for every strategy of $A$, player $B$ can play to make $E(g)<m_{x}-\varepsilon$.

For each possible next position, $y_{i}, i=1,2, \cdots, k$, let $f_{n_{i}}$ be such that $\operatorname{Val}_{y_{i}}\left(G_{f_{n_{i}}}\right)<m_{y_{i}}+\varepsilon / 2$. Let $m=\max _{i}\left(n_{i}\right)$; so that for all $i$,

$$
\operatorname{Val}_{y_{i}}\left(G_{f_{m}}\right)<m_{y_{i}}+\varepsilon / 2 \text {. }
$$

Now for any fixed strategy of player $A$, let $B$ play according to the assumption, to make $E(g)<m_{x}-\varepsilon$ and then play $\varepsilon / 2$ optimally in $G_{f_{m}}$ to make

$$
\begin{aligned}
E_{x}\left(f_{m}\right) & \left.\leqq \sum_{i=1}^{k} p\left(y_{i}\right) \operatorname{Val}_{y_{i}}\left(G_{f_{m}}\right)+\varepsilon / 2<\sum_{i=1}^{k} p\left(y_{i}\right) m_{y_{i}}+\varepsilon \quad \text { (by }(1)\right) \\
& =E(g)+\varepsilon<m_{x}
\end{aligned}
$$

(by assumption) which contradicts the fact that $m_{x}=\lim _{n} \operatorname{Val}_{x}\left(G_{f_{n}}\right)$, and the lemma is proved.

Now we are ready for the

Proof of Theorem 2.1. We shall show that for fixed $\varepsilon>0, A$ can guarantee that $E(f) \geqq \lim _{n} \operatorname{Val}\left(G_{f_{n}}\right)-\varepsilon=m_{e}-\varepsilon$ (where $e$ denotes the empty sequence). This will complete the proof, since $\left\{\operatorname{Val}\left(G_{f_{n}}\right)\right\}$ is a nonincreasing sequence, and so $U\left(G_{f}\right) \leqq \lim _{n} \operatorname{Val}\left(G_{f_{n}}\right)$.

First, let $A$ play optimally in $G_{e}^{*}$, and then, if $x_{n}$ is the position after the $n$th move, let $A$ play optimally in $G_{x_{n}}^{*}$. Define the random variables $X_{0}=m_{e}$; if $n \geqq 1, X_{n}=m_{x_{n}}$ if $x_{n}$ is the position after the first $n$ moves. By the lemma, we have $E\left(X_{n} \mid X_{n-1} \cdots X_{0}\right) \geqq X_{n-1} \Rightarrow$

$$
E\left(X_{n}\right) \geqq m_{e}
$$

for all $n$.

Now, using the usual facts about upper semicontinuity, for fixed $k$ (if $z=\left(z_{1}, z_{2}, \cdots\right)$ is the resulting sequence of moves), there exists 
$N_{(k, z, \varepsilon)}$ such that if $n \geqq N_{(k, z, \varepsilon)}$, any sequence $\omega=\left(\omega_{1}, \omega_{2}, \cdots\right)$ agreeing with $z$ up to the $n$th move has the property

$$
\begin{aligned}
f_{k}(\omega)<f_{k}(z)+\varepsilon & \Rightarrow \operatorname{Val}_{\left(z_{1}, z_{2}, \ldots, z_{n}\right)}\left(G_{f_{k}}\right)<f_{k}(z)+\varepsilon \\
& \Rightarrow m_{\left(z_{1}, z_{2}, \ldots, z_{n}\right)<f_{k}(z)+\varepsilon} \\
& \Rightarrow \text { for all } z, \lim \sup _{n} X_{n}(z)<f_{k}(z)+\varepsilon \\
& \Rightarrow \text { (by Fatou) } \lim \sup _{n} E\left(X_{n}\right)<E\left(f_{k}\right)+\varepsilon \\
& \Rightarrow E\left(f_{k}\right)>m_{e}-\varepsilon \text { for all } k \\
& \Rightarrow E(f)>m_{e}-\varepsilon
\end{aligned}
$$

(by the dominated convergence theorem).

COROLLARY 1. If $f_{n}$ are lower semicontinuous, $f_{n} \uparrow f$, then $\lim _{n} \operatorname{Val}\left(G_{f_{n}}\right)=$ $\operatorname{Val}\left(G_{f}\right)$

Proof. The negative of an u.s.c. function is l.s.c. so the theorem applies by reversing the roles of the players.

COROllaRy 2. Games with lower semicontinuous payoff functions can be approximated by finite games.

Proof. Suppose $f$ is 1.s.c. Define $f_{n}$ by $f_{n}(v)=\inf _{\omega \in S} f(\omega)$ where $S=\{\omega \in \Omega \mid 1$ st $n$ coordinates of $\omega$ agree with the 1st $n$ coordinates of $\nu\}$. Then the games $G_{f_{n}}$ are "finite", since the payoff is decided in the first $n$ moves. But the fact that $f$ is l.s.c. implies $f_{n} \uparrow f$, so we just apply Corollary 1 . (The functions $f_{n}$ are continuous.)

COROllary 3. Open games can be approximated by finite games, i.e., if $f=I_{\mathbb{C}}$ where $\mathcal{O}$ is an open set (in the product topology on $\Omega$ ) then the game $G$ can be approximated by the games $G_{n}$, where the payoff in $G_{n}$ is 1 if $\mathcal{O}$ is hit by the nth move, 0 otherwise. (This is actually a special case of Corollary 2.)

Proof. Immediate since $I_{\mathscr{O}}$ is l.s.c.

A CountereXAmPle. Approximation theorems do not exist in general as the following example shows. Let $A_{n}=B_{n}=\{0,1\}$ for $n=1,2, \cdots$, so $\Omega=\prod_{n=1}^{\infty}\{0,1\} \times\{0,1\}$. Let $S_{n}=F_{n} \cup G$ where $F_{n}=\{\omega \in \Omega \mid \exists i \leqq n$ with $\left.\omega_{i}=(1,1)\right\}$ (in other words $F_{n}=\{\omega \mid$ both players say 1 on the same move sometime before the $n$th move $\}$ ), and $G=\{\omega \in \Omega$ (player $B$ says 0 on every move\}. Clearly $F_{n}$ and $G$ are closed sets, so the functions $I_{S_{n}}$ are upper semicontinuous. Now the games $G_{n}$ with payoffs $I_{S_{n}}$ have value 0 since player $B$ need only say 0 for the first $n$ moves and 1 sometime after that to keep play from hitting $S_{n}$. Also since $S_{n+1} \supset S_{n}$ for all $n, I_{S_{n}} \uparrow I_{S}$ where $S=\bigcup_{n=1}^{\infty} S_{n}$. But the game with payoff $I_{S}$ has value 1 which player $A$ 
can achieve by merely saying 1 on every move. Player $B$ either must say 0 every time or 1 sometime and so $S$ is hit.

AN OPEN QUESTION. We do not know whether if $f_{n}$ are continuous, $f_{n} \rightarrow f$, then $\operatorname{Val} G\left(F_{n}\right) \rightarrow \operatorname{Val} G(F)$. This question has some relevance to the study of stochastic games (see [2], [3]).

I wish to express my gratitude to David Blackwell for many helpful conversations during the course of this research.

\section{REFERENCES}

1. D. Blackwell, Infinite $G_{\delta}$-games with imperfect information, Zastos. Mat. 10 (1969), 99-101. MR 39 \#5158.

2. D. Blackwell and T. S. Ferguson, The big match, Ann. Math. Statist. 39 (1968), 159-163. MR 36 \#6211.

3. D. Gillette, Stochastic games with zero stop probabilities, Contributions to the Theory of Games, vol. 3, Ann. of Math. Studies, no. 39, Princeton Univ. Press, Princeton, N.J., 1957, pp. 179-187. MR 19, 1147.

4. J. W. Milnor and L. S. Shapley, On games of survival, Contributions to the Theory of Games, vol. 3, Ann. of Math. Studies, no. 39, Princeton Univ. Press, Princeton, N.J., 1957, pp. 15-45. MR 19, 1147.

5. M. Sion, On general minimax theorems, Pacific J. Math. 8 (1958), 171-176. MR $20 \# 3506$.

Department of Mathematics, Case Western Reserve University, Cleveland, OHIO 44106 